\title{
The Changing Nature of Clinical Decision Support Systems: a Focus on Consumers, Genomics, Public Health and Decision Safety
}

\author{
E. Coiera, A. Y. S. Lau, G. Tsafnat, V. Sintchenko, F. Magrabi \\ Centre for Health Informatics, University of New South Wales, Sydney, Australia
}

\begin{abstract}
Summary
Objectives: To review the recent research literature in clinical decision support systems (CDSS).

Methods: A review of recent literature was undertaken, focussing on CDSS evaluation, consumers and public health, the impact of translational bioinformatics on CDSS design, and CDSS safety.

Results: In recent years, researchers have concentrated much less on the development of decision technologies, and have focussed more on the impact of CDSS in the clinical world. Recent work highlights that traditional process measures of CDSS effectiveness, such as document relevance are poor proxy measures for decision outcomes. Measuring the dynamics of decision making, for example via decision velocity, may produce a more accurate picture of effectiveness. Another trend is the broadening of user base for CDSS beyond front line clinicians. Consumers are now a major focus for biomedical informatics, as are public health officials, tasked with detecting and managing disease outbreaks at a health system, rather than individual patient level. Bioinformatics is also changing the nature of CDSS. Apart from personalisation of therapy recommendations, translational bioinformatics is creating new challenges in the interpretation of the meaning of genetic data. Finally, there is much recent interest in the safety and effectiveness of computerised physician order entry ( (POE) systems, given that prescribing and administration errors are a significant cause of morbidity and mortality. Of note, there is still much controversy surrounding the contention that poorly designed, implemented or used CDSS may actually lead to harm.

Conclusions: CDSS research remains an active and evolving area of research, as CDSS penetrate more widely beyond their traditional domain into consumer decision support, and as decisions become more complex, for example by involving sequence level genetic data.
\end{abstract}

Keywords

Decision support systems, consumer informatics, translational bioinformatics, biosuvveillance, safety

Yearb Med Inform 2009:84-95

\section{Introduction}

Clinical decision support systems (CDSS) have developed over the last 50 years as tools that assist clinicians, mainly doctors, with a number of standard decision tasks, such as formulation of a diagnosis, prescription of a medication, interpretation of a diagnostic result, or therapy planning (see Box 1). In recent years, researchers have concentrated less on the development of novel technologies, which carry out some or all of the reasoning associated with such tasks, and have focussed more on the impact of CDSS in the working clinical world.

This paper begins with a review of changes in the way CDSS are evaluated, with a focus on understanding their real world impact. We next highlight another significant trend in CDSS research, which is the broadening of the intended user base beyond front line clinicians. Consumers, for example, are now a major focus for biomedical informatics, as it is clear that the emergence of engaged and informed consumers should lead to a more effective health system [1], with associated improved health outcomes - although that prospect itself remains a goal rather than proven certainty. Public health officials, tasked with detecting and then managing disease outbreaks at a health system, not individual patient level, are also increasingly the focus of new CDSS developments, and are also reviewed here.
Bioinformatics is moving out from the laboratory bench and changing the nature of CDSS in some subtle and not so subtle ways. Apart from the obvious personalisation of therapy recommendations possible by knowing an individual's genotype, allowing more effective selection of medications for example, translational bioinformatics is creating new challenges in the interpretation of the meaning of genetic data.

There is much recent interest in the safety and effectiveness of computerised physician order entry (CPOE) systems, given that prescribing and administration errors are a significant cause of morbidity and mortality in many health systems. Of note, there is still much controversy surrounding the contention that poorly designed, implemented or used CDSS may actually lead to harm, and this remains an ongoing theme in the recent research literature [2]. We complete this review with an update of the recent literature exploring CDSS safety. The review is not exhaustive, and does not cover progress in high data volume domains such as intensive care, the operating room, and emergency. For example, alerts and alarms on continuously monitored patients are both an important CDSS application area and also one where a great deal of data are available for building recognition and prediction systems. 


\section{Box 1 Clinical Decision Support Systems}

CDSS store clinical knowledge, usually about a very specifically defined task, and typically reason with patient data to generate patientspecific inferences. There are many different types of clinical task to which CDSS can be applied [3] including:

Alerts and reminders. A CDSS attached to a patient monitoring device like an ECG or pulse oximeter can warn of changes in a patient's condition or may scan laboratory test results, drug or test order, or the electronic health record (EHR) and send reminders or warnings. Reminder systems notify clinicians of important tasks that need to be done. For example, an outpatient clinic reminder system may highlight patients requiring immunizations on the day's list.

Diagnostic assistance. When a case is complex, rare or the dinician making the diagnosis is inexperienced, CDSS can help formulate likely diagnoses based on patient data, and the system's understanding of illness stored in its knowledge base. Diagnostic assistance is often needed with complex data, rare presentations of common illnesses or diagnoses that require specialised expertise, and have an important educational role.

Therapy critiquing and planning. Critiquing systems look for inconsistencies, errors and omissions in an existing treatment plan. Planning systems have more knowledge about the structure of treatment protocols and can be used to formulate a plan based upon patient data and treatment guidelines.

Prescribing decision support systems check for drug-drug interactions, dosage errors, and if connected to an EMR, for other prescribing contraindications such as allergy. They may offer other benefits like automated script generation and electronic transmission of the script to a pharmacy.

Information retrievalsystems can assist in formulating appropriately specific and accurate clinical questions, can act as information filters, by reducing the number of documents found in response to a query to a Web search engine, and can assist in identifying the most appropriate sources of evidence appropriate to a dinical question. 'Infobuttons' retrieve information related to a specific context e.g. within a patient's electronic record.

Image recognition and interpretation. Many clinical images are automatically interpreted, from plane $X$-rays through to more complex images like angiograms, (T and MRI scans. This is of value in mass-screenings, for example, when the system can flag potentially abnormal images for detailed human attention.

\section{Advances in Benchmarking the Impact of Decision Support}

Much recent attention in biomedical informatics focuses on the relatively low adoption rates of technologies such as CDSS and seeks to understand why apparently well-designed systems, which perform well on traditional measures, are not perceived to be useful by clinicians. It may be the case that we need to formulate a broader set of metrics, more closely associated with clinical practice, to better understand what constitutes an effective CDSS. The evaluation of CDSS typically occur at three distinct points:

1 - Process metrics judge a CDSS by its capability to satisfy an intermediate goal. For example, the effectiveness of an information retrieval system may be measured by the number of relevant documents it retrieves, which is assumed to be a proxy measure for an effective decision outcome;

2 - Decision outcome metrics judge a CDSS by the quality of decisions made, for example by measuring the number of correct answers clinicians make, such as appropriate prescriptions, aided by the CDSS and unaided;

3 - Clinical outcome measures judge a CDSS by the ultimate impact it has on clinical processes, such as clinical outcomes like infection rates, death rates or length of stay in hospital.

Several recent research results underscore the challenge of CDSS evaluation. Document relevance, for example, is considered a cornerstone metric for the evaluation of information retrieval systems [4], and this metric is widely used when assessing the effectiveness of clinical systems designed to assist decision-making by identifying the most relevant evidence (e.g. [5]). It is surprising however that until recently no attempt has been made to test whether this proxy process measure actually correlates with changes in decision or clinical outcomes. One recent laboratory study we conducted looked at the decision outcomes of 227 health consumers and found that user relevance rankings had little or no predictive power [6]. Relevance rankings were unable to predict whether the user of a search engine could correctly answer a question after search and could not differentiate between two search engines with statistically different performance in the hands of users. Only when users had strong prior knowledge of the questions, and the decision task was of low complexity, did relevance appear to have modest predictive power. Studies such as this raise the concern that many well accepted process level measures, assumed to be a proxy for harder to measure outcomes, may not correlate well or at all with such outcomes. Therefore evaluators need to convince themselves that they are using well validated process metrics, rather than continuing with unvalidated traditional measures.

Focusing solely on decision outcomes, like the ability to arrive at a correct decision, may also be obscuring significant CDSS benefits. For example, the search for clinical information often occurs in time-poor settings. Doctors may spend no more than two minutes searching for an answer [7] and often abandon searches when no answer is forthcoming [8]. Consequently, we have proposed that the rate at which clinicians are able to make decisions, their decision velocity, could be an important metric for benchmarking the performance of CDSS [9]. Analyzing the performance of 75 doctors and nurses on a battery of clinical decision tasks using two different systems, we were able to show that there was little statistical difference between the number of correct answers produced by the clinicians. However, when the dynamics of decision making were taken into account, one system was clearly superior, improving the decision velocity of its users over a two minute period, as well as resulting in more correct answers in the first 2 minutes of use. Consequently, it seemed likely that in time restricted clinical settings, a system which allows clinicians to arrive at a correct decision more quickly is likely to have a greater impact on clinical 
outcomes. This is likely because fewer clinicians would abandon the CDSS because of time constrains, and revert to their unaided judgement. By using decision metrics which better reflect the needs and pressures of the clinical work place, it is likely that we will be able to identify which CDSS are more likely to have an effect in the real world, as opposed to the evaluation bench.

\section{Consumer Decision Support}

Decision support systems (DSS) for healthcare consumers, sometimes known as decision aids, are designed to help people make informed choices between different options to manage their health [10]. While DSS such as online search tools can improve the quality of consumer decisions [11], uncertainty and anxiety, personal values, preferences and emotions can confound the consumer decision-making process, often intensified by decision complexity and cognitive biases [12]. DSS are thus thought to be most useful for consumers facing complex decisions, such as tradeoffs between short and long term outcomes, major differences between options, or when choices can lead to grave outcomes [13, 14].

\section{Development of Consumer Decision} Support Systems

Consumer DSS vary widely and are available in various forms of media. Early consumer DSS ranged from home library reference databases and body atlases to patient advice and handouts [15]. Later forms began to be delivered on the Internet, with the more prominent systems emerging in the 1990s, including websites like the Comprehensive Health Enhancement Support System (CHESS) [16], Database of Individual Patient Experiences (DIPEx) [17], Foundation for Informed Medical Decision Making (FIMDM) [18], Mayo Clinic [19] and NexCura [20]. These websites offered consumers detailed information about treatment options for a range of health topics and sometimes provided them with aids to select between management options. However, a review of online consumer DSS reported that none of these systems scored comprehensively against four core criteria i.e. access to other patients' experiences, online community network, user-tailored prognostic outcomes and free public access [21].

Today, online consumer DSS may take many forms and are developing rapidly. These include (i) information therapies (e.g. self-administered tools to manage anxiety or depression [22]) and self-care tools, (ii) healthcare consumer virtual communities, (iii) online decision support and information retrieval systems, (iv) personal health record and management systems, (v) clinician-patient communication tools, and (vi) e-health services, such as transactional-based services (drug refills and appointment scheduling) and carerelated services (medical and medication advice) [23].

Systems reported in the research literature include MyGroupHealth [24], PatientsLikeMe [25], PatientSite from the Beth Israel Deaconess Medical Center [26] and PAMFOnline from the Palo Alto Medical Foundation [27]. In particular, initiatives and organisations such as the International Medical Informatics Association (IMIA) Web 2.0 taskforce [28] and the International Society for Research on Internet Interventions (ISRII) [29] are exploring the use of Web technologies for the development of online tools for healthcare consumers [30].

\section{Review of Consumer Decision Support} System Research

Consumer aids and DSS have begun to be studied extensively in the past decade. O'Connor and colleagues from the Ottawa Health Research Institute, for example, proposed a systematic framework for designing decision aids. Built on concepts from psychology, social psychology, economics and social support, the Ottawa Framework of Decision Support contains a series of workbooks, tutorials and principles to guide a designer to ensure that the content in a decision aid reflects patients' values and is evidence-based with policies in place to update and maintain it [31].

To verify whether decision aids are built to an internationally-recognised set of criteria, O'Connor and Elwyn led the International Patient Decision Aid Standards (IPDAS) Collaboration to set up a quality framework for patient decision aids [32]. IPDAS has brought together stakeholders from 14 countries to develop 12 categories of quality measures for consumer DSS, which are now incorporated in their proposed standard [33]. A Cochrane Decision Aid Registry has also been established to record decision aids and their evaluation results; so far, the registry contains over 200 decision aids, covering a wide range of health topics related to treatment and screening decisions [34].

One of the most consistent benefits of using decision aids is increased consumer knowledge of management options and likely outcomes [35]. Consumers feel more supported and participate more actively in their healthcare decision-making process when they use decision aids [13]. A Cochrane review found that patients using decision aids experience less uncertainty when facing treatment and diagnosis decisions compared to those who did not use them [10]. It also found that using decision aids reduces the number of patients remaining undecided and improves the concurrence between patients' values and their choice [10]. A recent randomised controlled trial with 611 men considering prostate cancer screening found that using an online decision support aid reduced their uncertainty and increased their knowledge of prostate cancer compared to providing participants with links to health websites [36].

Consequently, reducing decisional conflict (i.e. the uncertainty about which course of action to take when given choice among competing actions) [37], is a central goal in the design and evaluation of consumer DSS. The Conflict Theory of Decision Making (CTM) postulates that how one copes during the process of making a decision (e.g. 
procrastinates, ignores information, uncritically adopts a new course of action, painstakingly searches for relevant information, or panics), is dependent on whether one is aware of a serious risk if nothing is done, has hope in finding a better alternative, or believes that there is enough time to learn about and assess the situation to choose a better alternative [38]. Based on this theory, the Decisional Conflict Scale (DCS) can measure an individual's level of uncertainty when making a healthcare decision [37], and is now widely used in consumer decision-making research. For example, a cross-sectional national survey conducted with 635 Canadians measuring their levels of decisional conflict when facing complex healthcare decisions revealed a detailed picture of consumers' healthcare decision-making needs [39]. Consumers manifested decisional conflict in different ways, ranging from verbalising uncertainty about what to do, wanting to delay a decision, to wavering between available choices.

Many factors can contribute to decisional conflict. Information deficits are considered the most frequent factor (e.g. lack of information regarding options, pros and cons and their likelihood), followed by social deficits (e.g. not knowing what others decide or recommend, receiving insufficient support), then cognitive deficits (e.g. lack of skills and ability to make this type of decision), and finally the emotions involved in the decision-making process. Consumers in the Canadian crosssectional survey also used different strategies to make complex healthcare decisions [39]. For example, almost three-quarters sought information and considered how important each of their options were (i.e. information sources), and more than half sought support and information on what others decided or recommended (i.e. social sources). Additionally, there was an association between the type of decision to be made, a consumer's perceived level of comfort and their preferred channel of seeking information and support. For example, those making decisions about institutionalising a family member were more likely to feel distressed and seek support from other people than those making decisions about birth control.

Decision biases provide a further confounding factor in the decision making task. It has been understood for quite some time that humans give different weights to the information presented to them, for example favouring information that confirms their own prior beliefs, or information that comes first or last in a sequence. The existence of such decision biases is exploited in modern marketing to the advantage of commercial enterprises, but can also lead to poor quality decision-making. Studying the impact of decision biases on health decisions after internet search, experiments seem to show that person's prior belief (anchoring), accessing documents at different positions in a list of search results (order effect), reading documents for different lengths of time (exposure effect) have a significant impact on post-search decisions, independently of the content itself [40]. Recent work also shows that well-designed user interfaces are able to moderate the impact of such biases on consumer health decisions [41], offering hope that well designed online tools can significantly enhance the quality of consumer health decisions.

Future Directions of Consumer Decision Support System Research

Over a decade of research work has gone into developing an evidence-based approach to designing and creating the content in consumer DSS. However, there has not been as much research into the strategies needed to present this content to consumers, nor in studying consumers' decision-making needs as their health issues progress over time [10]. At the same time, Web technologies continue to develop rapidly, and commercial developers seem to be building online applications for consumers at a faster rate than e-health researchers can anticipate. This presents a number of challenges for researchers working in the online consumer DSS space:
- Safety of online consumer DSS: When designing online consumer DSS, how do we craft content so that consumers are not misled into taking significant actions without medical supervision? Further, as obtaining advice and recommendations from social sources plays an important part in consumers' decisionmaking process, how can we promote a safety culture amongst members of online communities so that members refrain from offering advice that leads to medically-unsupervised actions? Recent research shows that consumers who are least confident in their decisions are the ones most likely to be swayed by social feedback [11]. Consequently we need to understand how those most vulnerable to undue influence are best supported when entering online communities.

- Privacy in online consumer DSS: How can we ensure that the privacy of personal data or decisions is preserved, striking the right balance between encouraging consumers to share their experiences and risking the privacy of their personal health data being exposed and exploited? Further, how can we ensure that members of an online community are genuine healthcare consumers, and exclude those with different goals from participating or lurking in the community?

- Search, social and decisional conflict: As information and social deficits seem to play a major role in generating decisional conflict, how can we innovatively use Web search and social technologies to reduce decisional conflict when consumers face complex healthcare decisions?

- Personal health records and health services: How can we develop integrated systems that incorporate personal health records (PHRs), decision support, information therapies, online self-care management, and other services? How can consumer DSS make use of the emerging PHR infrastructure to personalise decision 
support for consumers? Furthermore, as online consumer DSS developed by researchers have to compete with tools created by commercial entities, not all of which will be built using the best evidence available, how do we guide consumers to avoid using tools that are not sound?

- Web 2.0 and interaction design: Besides providing consumers with quality content online, how can we innovatively design and/or apply Web 2.0 technologies to enrich consumers' interaction online, as well as optimise the distribution of consumer DSS to those in need?

- Health behaviours: What consumer behaviours are we seeing emerge as consumers adopt online tools to manage their health, and what are the outcomes of such behaviours? For example, how does the relationship between patients and clinicians alter as patients access previously unavailable information [42]? What additional support will consumers now need as their decision-making role extends more widely across the healthcare journey?

Future research efforts in consumer DSS should thus focus on optimising the design of online interaction models, integrating Web search, social and e-health technologies and providing consumers with tailored information, support and tools. In particular, emerging issues relating to safety and privacy online need to be addressed. With continuing commercial and public investment in consumer e-health systems, supported by a solid research base, we can anticipate that there will be a significant uptake of consumer DSS, with growing numbers of consumers feeling empowered to make choices about their healthcare.

\section{Translational Bioinformatics and Clinical Decision Support}

Translational bioinformatics is the application of post-genomic bioinformatics tools and techniques to the study of human disease and improving hu- man health [43]. Thus far, the penetration of genetic methods in clinical practice has largely been limited to screening for diseases [44]. Surprisingly, the growth in availability of public genomic data $[45,46]$ and testing technologies have not yet resulted in the expected surge in uptake of genetic tools in clinical practice. The most likely explanation for the poor penetration of genomic medicine to date is the lack of "genetic literacy" or preparedness amongst practitioners [44, 47-50]. At the same time, more recent developments from the research community are beginning to provide tools for a far broader range of clinical tasks, including pharmacogenomic methods to identify the most appropriate therapy or dose for individuals based upon their genetic make-up.

Bioinformatics data is different in many ways from the sources clinicians are familiar with, such as guidelines, systematic reviews, and prescriptionbased prompts, alerts and reminders. Genetic data can be voluminous, but also often significantly more ambiguous than traditional data. It is now becoming clear that there are multiple polymorphisms in human DNA that can produce the same broad clinical syndrome or disease. For example, analysis of large B-cell lymphoma using DNA microarray technology has identified distinct clusters of patients, with dramatically different mortality rates [51]. Where there are large groups of patients with similar genetic profiles and the connection to disease is well mapped, then the situation is straightforward, but in cases where an individual's genetic sequence is rare, or the association between gene and disease is associational, or multifactorial, then knowledge of genetic sequence alone is insufficient to understand the implications of DNA information for disease [3]. Consumers and clinicians can be faced with a raft of polymorphisms in an otherwise well individual's genome, using even currently limited testing regimes, with many of them linked in some way with different diseases. This "disappearing cohort" problem means that the more specific the information we have about a patient, the harder it will be to find a matching cohort study [52].

Counselling on such a basis is thus likely to be challenging, until there is a better understanding of the meaning of such variants. The growth of DNA data is indeed well ahead of our understanding of its implications for individual health. In such an environment, our reliance on biological theory and simulation will increase in contrast to the paradigm of evidence based medicine.

Warfarin (Coumadin) Dose Maintenance Warfarin dosing provides us with an early example of the widespread use of genetic information to support prescribing and suitable for decision support. Warfarin is a blood anticoagulant with a dose requirement that depends on body mass as well as genetic factors [53]. Incorrect dosing can result in severe adverse effects: too small a dose and the drug is ineffective, too high a dose can lead to haemorrhage [54]. The process of arriving at a patient-specific dosage is long and typically requires several adjustments and blood tests $[55,56]$.

It is now known that genetic variants of the enzyme that metabolizes warfarin, cytochrome P-450 2C9 (CYP2C9), and of a key pharmacologic target of warfarin, vitamin $\mathrm{K}$ epoxide reductase (VKORC1), contribute to differences in responses to warfarin doses [57]. Genetic testing is thus likely to improve therapy by better matching dosing regime to the individual [58]. In August 2007 the US FDA approved a significant change in labelling rules for warfarin to include genetic testing as part of the dose selection process [59]. This change in legislation is expected to reduce the number of adjustments required to reach an optimal dose and lead to an expected savings of $\$ 1.1$ billion annually in the US [60].

Since warfarin prescription is relatively simple, and does not require the prescribing clinician to have much "genetic literacy", several commercial decision support systems exist to support 
the process (e.g. http://www. warfarindosing.org/Source/Home.aspx and http://www.pace-med-apps.com/ CoumCalc.htm). It is also likely that the genetic basis for variations in response to warfarin will become better understood, and that the initial simplicity of gene-dose models will rapidly be replaced by a more complex set of relationships, making it essential to use a CDSS to match genotype to dose.

\section{Antimicrobial Drug Selection}

Whilst warfarin presents us with an example of how genetic data can support tasks in the more traditional CDSS space, antibiotic prescribing provides us an example of the complexities associated with some genetic domains. Antimicrobial drug resistance is widely recognized as a global public health threat with major economic impact [61, 62] and leading to a doubling in hospital mortality [63, 64]. Antibiotic resistance compromises the treatment of serious infections, especially in the critically ill, and the continuing emergence of multi-drug resistance threatens to produce strains against which no drugs are effective.

Computational systems that support antibiotic prescription systems [65-67] have in the past typically used heuristic rules to find a drug that the infecting organism is most likely to be sensitive to, and that also minimises the chances of encouraging drug resistance. Such CDSS have been shown to improve antibiotic prescription with regards to both ecological impact and patient outcome [66-68].

However, none of these systems use microbial genetic information [69], which may allow a richer categorization of the likelihood of disease virulence and drug resistance. There are hundreds of known antibiotic resistance genes [70] and GenBank contains thousands of entries in which they are sequenced. Knowing the genetic profile of an infecting organism should thus guide the selection of therapy against it. Antibiotic selection using genetic data is however complicated by hori- zontal gene transfer between organisms, supported by a battery of different molecular mechanisms by which bacteria can spread genes amongst themselves, and even across species. The clinical problem is further complicated by geographic location and the recent history in that location, as different populations of organisms can be found at different locations, but cause similar disease. Consequently, even as new tests like rapid PCR promise to identify some genetic traits of infecting organisms, it is likely that the high mobility of genetic elements, and their continuing evolution, will mean that any CDSS will often have to fall back on probabilistic models of the likelihood that particular genes are present. Similar challenges exist when building pathogen profiles in support of public health decision-making (see next section).

\section{Future Directions}

Looking at the next decade two related trends are evident: 1 - genomic literacy will be an increasingly important skill for clinicians; and 2 - advances in biological theory, mediated via bioinformatics and genetic medicine, will lead to clinical tasks needing cognitively richer forms of decision support than currently provided. Consequently CDSS system designers will need to develop a new set of task models and interaction designs to support these genetically supported clinical tasks. CDSS that reason about genomic data are also likely to use data from a variety of sources including textual, measured and even simulation results. Borrowing the MultiModelling approach from Systems Engineering, the knowledge bases of such CDSS may consist of several components (models), representing different data sources, biological processes and health system aspects, and a set of modelled interactions for those components. CDSS for translational bioinformatics are likely to thus take two broad forms - those that fit neatly behind the scenes of current tasks (e.g. Warfarin dosing) and those that support new tasks for clinicians (finding the 'vanishing' cohort that matches a patient's gene test results).

\section{Decision Support Systems for Public Health Surveillance}

The value of real-time decision support in areas of public health surveillance and infection control is becoming increasingly recognised [71]. CDSS can assist in the rapid identification of outbreaks and must be sufficiently sensitive to alert public health professionals early in the outbreak, when the increased incidence above baseline may not be detectable by manual surveillance. This involves achieving a balance between sensitivity and specificity as systems that identify very small clusters of diseases might provide overly frequent and unnecessary alerts.

\section{New Data Types and Applications}

Real-time linkage between emergency departments and public health provided information can enhance outbreak detection and response. There has been a stream of post-implementation evaluation projects reporting the performance of biosurveillance systems and their impact on public health decisions in different settings. Surveillance systems implemented in Northern America [72], Europe [73], Asia [74] and Australia [75] have proved useful for monitoring seasonal influenza and foodborne outbreaks in diverse emergent or disaster situations and mass-gatherings. These systems have been successfully employed to provide medical record and syndromic data monitoring for high-visibility events, such as the Olympic Games [76, 77], or for early warning for outbreaks in endemic areas for military forces [78].

Assessment of the ability of biosurveillance systems to detect potential bioterrorist attacks has been especially challenging and has relied on simulation or quasi-experimental studies. A range of niche applications based on statistical and machine learning methods have been developed for outbreak detection [75, 79-81] and examined in simulations of non-endemic bioterror 
attacks (e.g. the detection of cases of a windborne anthrax release) [81].

Electronic surveillance using webbased tools can improve the detection of outbreaks of infectious diseases. For example, the HealthMap system uses text-processing algorithms to query, integrate and visualize unstructured reports from news media, expert-curated accounts and official alerts on disease outbreaks in many languages [82, 83]. It can identify important disease outbreaks with $84 \%$ accuracy and provides an open resource for the global public health community.

Further work is required to refine syndrome definitions, improve data quality, and optimise sensitivity and specificity for anomaly detection and alerting. A unified model of aberrancydetection algorithms and a software infrastructure has been developed to enable the evaluation of CDSS performance. Buckeridge et al. [79] have used a task-analytic methodology to identify the common features and meaningful distinctions among different algorithms and to gather evidence about the relative performance of these algorithms.

Redesigning Health Records to Support Public Health and Data Integration CDSS in biosurveillance are often built as data integrators and data visualisation tools. Progress in high performance computing, Web 2.0 technologies and grid computing, as well as the development of new health care delivery models (e.g. telecare) have fuelled the development of such "systems of systems" integrating multiple data sources of varying data format and quality [84]. For example, spatiotemporal analysis of telehealth data, specifically fever calls, provided a timely and unique description of the evolution of a national influenza outbreak. Such tools may be used for tracking other diseases, although the lack of consistent comparison data makes this more difficult to assess [85].

Adapting current EHR systems to serve public health needs offers new opportunities for public health practice and policy as well as for patients and health- care providers [86]. For clinicians, a public health oriented EHR system could reduce the paperwork burden of communicable disease notification and provide decision support about community trends that could aid in diagnosis and treatment selection of individual patients. Automated reporting has the potential to improve the completeness of notification and compliance with quality assurance criteria [86]. In order to support public health, EHR data models would need to be expanded to incorporate additional environmental and non-medical data elements.

\section{Applying Genomics Data to Improve Public Health Outcomes}

High throughput sequencing is currently taking the biomedical research community by storm and there is a growing interest in building decision aids to reduce the complexity of genomic analyses for public health professionals and clinicians. Specifically, systems are being developed to assist in the matching of microbial sequences for public health investigations [87, 88]; to combine genetic and geographic data of a pathogen; to reconstruct its history; and to identify the migration routes through which the strains spread regionally and internationally [89]. The utility of matching pathogen finger- prints goes beyond specific questions related to the investigation of possible outbreaks. It can also be used for disease monitoring, by enabling a better understanding of the transmission of infection and the association between microbial types and clinical outcomes. Some examples of decision support systems based on the genomic profiling of pathogens with epidemic potential are listed in Table 1.

Multidimensional Data Analysis: Spatiotemporal Analysis for Public Health Intelligence

Spatial surveillance using geographic information systems (GIS) can enhance the likelihood that even localized events will be detected, and their extent measured in space and time. The output from these systems ultimately needs to be integrated into the clinical and diagnostic process. Temporal and spatial clustering and GIS applications have significantly enhanced the utility of decision support tools for public health. They have become routine aids in many electronic communicable disease notification systems. Automated, realtime public health surveillance systems linking emergency departments and public health units similar to the Automated Epidemiological Geotemporal Integrated Surveillance (AEGIS) sys-

Table 1 Examples of computerised decision support based on molecular fingerprinting of pathogens with epidemic potential

\begin{tabular}{|l|l|l|l|l|}
\hline End users & Decision task & Pathogen & Software tools & References \\
\hline $\begin{array}{l}\text { Publichealth } \\
\text { professionals }\end{array}$ & $\begin{array}{l}\text { Monitoring of } \\
\text { epidemiological trends, } \\
\text { biothreat assessment, surge } \\
\text { capacity planning }\end{array}$ & $\begin{array}{l}\text { Multiple emerged } \\
\text { and emerging } \\
\text { viruses }\end{array}$ & HealthMap & $\begin{array}{l}\text { Brownstein, } \\
2008 \text { [83] } \\
\text { Davis, 2008 [77] }\end{array}$ \\
\hline Clinicians & $\begin{array}{l}\text { Interpretation of pathology } \\
\text { reports and prescribing of } \\
\text { antimicrobials }\end{array}$ & HIV & $\begin{array}{l}\text { genotypic } \\
\text { interpretation } \\
\text { systems } \\
\text { VircoType, ViroScore }\end{array}$ & Rosen-Zvi, 2008 [88] \\
\hline $\begin{array}{l}\text { Infection control } \\
\text { practitioners }\end{array}$ & $\begin{array}{l}\text { Lomparative analysis and } \\
\text { clustering of pathogens. } \\
\text { Detection of episodes of } \\
\text { recent transmission }\end{array}$ & $\begin{array}{l}\text { MRSA and other } \\
\text { hospital-acquired } \\
\text { microorganisms }\end{array}$ & Ridom StaphType & Mellman, 2006 [81] \\
\hline
\end{tabular}


tem have been employed in many jurisdictions in Northern America and Australia [91, 92]. The quality of data feeds and the lack of epidemiological feedback for alerts generated by these systems have remained the main challenges in their dissemination and uptake. However, recent studies have attempted to address these issues and to provide a scalable definition of outbreaks that can be used in the GIS applications [93].

Public health can also be of value for healthcare managers. They provide new types of data that can be invaluable in informing policy makers at the local, regional and national levels [84]. Biosurveillance CDSS should allow policymakers to use their own realistic baseline values and program projections to assess the relative impact of different interventions to improve the probability and timeliness of detecting clusters of emerging infections. Policymakers can also use results to target investments to improve their surveillance infrastructure [91].

\section{Safety of Clinical Decision Support Systems}

It is now widely recognised that, despite their manifest and manyfold benefits [94], CDSS can also generate new types of errors that are computer-related [2, 95, 96]. In 2008, the US Joint Commission on Accreditation of Healthcare Organizations (JCAHO) published a new Sentinel Events alert on Health IT signalling a pressing need to direct more efforts towards safety within design, implementation and use of CDSS amongst a range of supporting systems currently being used in clinical settings [97]. Although evidence about the overall risks associated with CDSS is scarce, available data suggest that computer related errors have the potential to pose a significant risk to patient safety. Computer errors relating to CDSS can be generally distinguished into two main classes: 1) machine errors and, 2) errors in the hands of users.

Machine errors: At a fundamental level, the safety of any CDSS is directly de- termined by the completeness and accuracy of its knowledge base. A second source of computer-generated errors arises from the internal procedures and logic used within a CDSS to match its clinical knowledge base with patient data to generate alerts. Historically much of the research exploring the safety CDSS, has focussed on identifying if systems are broadly acceptable to users, or whether there is variation in system function, independent of any evidencebased standard to make such an assessment. For example, Wang et al. undertook a broad study of alert features in 10 US primary care systems and found a significant variation in software functionality when assessed against 60 'expert' recommendations, of which 12 specifically related to prescribing alerts [98]. Other investigators usefully attempt to determine if errors occur with CDSS use. Within a laboratory setting a Canadian system was reported to detect only 4 out of 15 clinically significant interactions, and was silent for only $96 \%$ to 247 non-clinically significant drug-drug interaction pairs [99].

More recent research is attempting to resolve the different causes of machine error. A French study found that $46 \%$ of 613 overridden alerts about drug-drug interactions were false-positives [100]. Of these $40 \%$ were attributed to system error because the CDSS did not take into account the dose, route and timing of administration in making recommendations about a potential interaction. A relatively smaller proportion of false positives related to known interactions where evidence-based guidelines were available to show that benefits outweighed the risks. The accuracy of such information is also critical to CDSS safety. One US study found that $36 \%$ of CDSS recommendations about drug-allergy and high severity drug interactions were not justified on the basis of scientific evidence [101]. When surveyed about the adequacy of alerts, $70 \%$ of primary care physicians reported that alerts did not provide them with the information they needed most of the time [102]. Users of another US primary care system reported that only 1 in 9 prescribing alerts were useful [103]. Drug duplication alerts were considered less useful than drug interaction alerts, which may have more serious consequences.

Errors in the hands of users: It is now widely recognised that the safety of clinical software is as much a product of human as it is of machine. Busy clinicians will routinely disable or override computer advice in busy clinical settings. There is growing evidence for such errors of omission resulting from alert fatigue which is an expected consequence of systems with poor specificity e.g. high rates of non-serious and irrelevant alerts. Between $49 \%$ to $96 \%$ of drug safety alerts are dismissed or overridden by clinicians [104]. A US study across six primary care centres found that $72 \%$ of 291,890 overrides over 12-months related to critical drugdrug interactions [105]. In a VA setting clinicians overrode $87 \%$ of critical drug-drug and $81 \%$ of drug-allergy alerts, some of which were attributed to the use of a commercial system which was not adequately customised to local needs [106]. The long-term impact of such systems in generating new errors and their influence on clinicians' decision-making has yet to be systematically investigated.

Beyond the two main classes of computer error which particularly relate to clinical software and its use, the safety of CDSS, as is the case with any health IT system, is understood to be a property of the overall system within which these technologies are implemented and used [107]. A range of work process, cultural and organisational issues may contribute to new types of error [108]. For example, implementation of CDSS may often eliminate or shift human roles resulting in reduced verification and checking of medications and laboratory requests. Errors may also arise from unsafe workarounds if the CDSS knowledge base is not trusted by staff or not updated in a timely manner or inadequately customised to local work process requirements. 
Making Clinical Decision Support Systems Safer

CDSS are still not subject to any formal regulation in many countries [109]. The safety of these systems has now started to be addressed by a number of international efforts to improve the safety governance of clinical software. Among such efforts, the US Certification Commission for Healthcare IT has introduced specific requirements for CDSS within functionality criteria for hospital and ambulatory care systems [110]. The US Leapfrog Group, another voluntary program, has developed the CPOE Evaluation Tool which provides an assessment of the adequacy of decision support within hospital CPOE systems to provide alerts for high frequency serious prescribing errors [111]. In September 2008 the UK National Health Service, which took a lead role in embedding a safety management approach into their procurement processes, published simple developer guidelines for a broad range of safety features in electronic prescribing systems. Future versions are planned to cover drug-drug interaction checking and other decision support functions not included in the initial publication [112]. The International Organisation for Standardization, based upon a risk management approach, is developing standards for the construction, implementation and use of clinical software.

Beyond certification and standardisation, which will provide a minimal level of safety assurance, the need for continuous monitoring at a local organisational level, analogous to the post-marketing surveillance of drugs to monitor patients for unknown and unexpected side effects, is recommended to detect new types of errors which may invariably arise from implementation and use of CDSS in complex environments that characterised by high local variability [113]. Following completion of its first survey of $\mathrm{CPOE}$ for medications management involving 1200 hospitals, the US Leapfrog Group published a warning about the need for quality assurance during implementa- tion to ensure the safety and effectiveness of CDSS [111]. In addition to the recommendations for systems implementation published by the Leapfrog Group and JCAHO, Walker and colleagues [107] propose seven useful strategies for risk-management over the entire lifecycle of electronic health record systems which are also applicable to CDSS. As is the practice in other safety-critical industries overall safety management systems [114] supported by automated surveillance systems, which have the capacity to improve the efficiency and effectiveness in detecting general patient safety problems, will invariably be required at a local organisational level to monitor the ongoing safety of CDSS and related systems [113].

Despite the lack of clear evidence about mechanisms for causation of CDSS machine errors, or errors arising in the hands of users, some design strategies to minimise the occurrence and impact of errors associated with CDSS for CPOE have started to emerge, including:

1) Reducing alert fatigue by customising and prioritising CDSS recommendations: The overall number of alerts may be reduced by local customisation of rules for alerts based on large commercial knowledge bases which usually generate a large number of alerts. Organisations will often elect to design alerts for a subset of the original drug database covering only the most safety critical medications. The availability of tools as well as policy and procedures to support local customisation are critical to the effectiveness of this strategy [113].

To further reduce alert fatigue designers could consider smarter CDSS that can be trained manually or automatically to individual practice requirements akin to an email spam filter that can be gradually trained to remove irrelevant alerts. For instance, clinicians should be able to train their prescribing software to provide alerts only for the new medications in a script and ignore repeat medications in cases where a clinician has previously noted an alert and the patient is known to tolerate a particular drug combination. In a similar manner software can be trained to automatically tune its performance based on patterns of alerts overridden by clinicians. Other measures include prioritising alerts by severity, which has been shown to improve the effectiveness of CDSS in a hospital setting [115]. However, allowing clinicians to customise alerts [116] and receive noninterruptive recommendations [117], may not always be feasible strategy [118], nor effective.

2) Simplifying user interactions with CDSS: Clinician acceptance of alerts can also be improved through more user-friendly interactions. For example, by providing a pick list of common reasons in place of a free text entry, in cases where users must provide a reason for overriding an alert [115]. A range of formal usability engineering approaches are being applied to identify problematic interface features as well as error-prone interactions [119]. Cognitive models are another technique which can be used to quantitatively examine and simplify user interactions with a CDSS [120]. In a departure from conventional text-based presentation of recommendations, the use of graphics and icons to convey medications information has the potential to simplify interaction, reduce the time taken to read CDSS recommendations and generate fewer errors [121]. At a broader level data mining methods such as sequential pattern analysis and Markov chain models show much promise to identify ways people use CDSS interfaces, and maybe simplify interaction paths for complex or problematic tasks [122].

3) Examining overall safety of CDSS within context of use: Risk-analysis approaches may be useful during design as well as post-implementation to examine specific functionality of a CDSS within a local context. Techniques such as the Failure Modes, Effects and Criticality Analyses (FMECA) are now being applied to CDSS implementation 
and may be potentially useful in continuous monitoring of systems [123]. Another strategy to examine overall safety that may be increasingly explored as more data about CDSS errors becomes available, is the use of computational models combining systems dynamics and agent-based approaches [124].

Any approach to engineering safer CDSS is highly reliant on systematic examination of the safety of CDSS both on their own as well as in the hands of typical users to better understand the frequency as well as mechanisms for computer errors. At present there is scarce information about the causation of computer errors and this is likely to hamper efforts to make CDSS safer. As CDSS continue to proliferate in the clinical world and beyond the challenge of ensuring the safety of these systems through design, implementation as well as ongoing monitoring and maintenance will only become greater. Future directions in research will lie at the intersection of patient safety, systems safety engineering, psychology, and human factors to develop new approaches specifically tailored to addressing unknown risks posed by CDSS in the hands of an expanding user base within highly variable settings. Some key areas for future research include the development of better techniques to detect errors and their mechanisms; methods for designing safer user interfaces and interactions; risk-analyses and modelling techniques to examine and manage safety on an ongoing basis.

\section{Conclusion}

CDSS research remains an active and evolving area of research, as CDSS penetrate more widely beyond their traditional domain into consumer decision support, and as decisions become more complex, for example by involving sequence level genetic data. In the early years, CDSS research largely focussed on improving the core decision technologies, and while there will always be a place for better technology, our cur- rent challenges remain to find ways of enhancing the uptake of CDSS, in support of a broad range of healthcare goals. To do this, we find ourselves surprisingly weak in our understanding on how people actually use CDSS, and what benefits we can provide to those struggling with complex, challenging or personally threatening health decisions.

\section{Acknowledgments}

This research is supported in part by grants from the Australian Research Council (LP0775532, LP0667531 and DP0772487), a NSW Health Capacity Building Infrastructure Grant, the HCF Health and Medical Research foundation, and NHMRC Program Grant 568612.

\section{References}

1. Coiera E, Hovenga EJ. Building a sustainable health system. Yearb Med Inform 2007:11-8.

2. Coiera E, Westbrook J, Wyatt J. The safety and quality of decision support systems. Yearb Med Inform 2006:20-5.

3. Coiera E. Guide to Health Informatics: Hodder; 2003.

4. Mizzaro S. Relevance: The whole history. Journal of the American Society for Information Science 1997;48(9):810-32.

5. Lu Z, Kim W, Wilbur WJ. Evaluating Relevance Ranking Strategies for MEDLINE Retrieval. J Am Med Inform Assoc 2009 Jan-Feb;16(1):32-6.

6. Coiera EW, Vickland V. Is Relevance Relevant? User Relevance Ratings May Not Predict the Impact of Internet Search on Decision Outcomes. J Am Med Inform Assoc 2008 July 1, 2008;15(4):542-5.

7. Ely JW, Osheroff JA, Ebell MH, Bergus GR, Levy BT, Chambliss ML, et al. Analysis of questions asked by family doctors regarding patient care. BMJ 1999 August 7, 1999;319(7206):358-61.

8. Ely JW, Osheroff JA, Ebell MH, Chambliss ML, Vinson DC, Stevermer JJ, et al. Obstacles to answering doctors' questions about patient care with evidence: qualitative study. BMJ 2002 March 23, 2002;324(7339):710.

9. Coiera E, Westbrook JI, Rogers K. Clinical decision velocity is increased when meta-search filters enhance an evidence retrieval system. J Am Med Inform Assoc 2008 June 25, 2008:M2765.

10. O'Connor AM, Stacey D, Rovner D, Holmes-Rovner M, Tetroe J, Llewellyn-Thomas H, et al. Decision aids for people facing health treatment or screening decisions. Cochrane Database Syst Rev 2001(3):CD001431.

11. Lau AYS, Coiera EW. Impact of Web Searching and Social Feedback on Consumer Decision Making: A Prospective Online Experiment. J Med Internet Res 2008;10(1):e2.

12. Arkes HR. The Psychology of Patient Decision Making. 2008 [12 Nov 2008]; Available from: http://symptomresearch.nih.gov/chapter_4/ index.htm.

13. O'Connor A. Using patient decision aids to promote evidence-based decision making. ACP J Club 2001 Jul-Aug:135(1):A11-2.

14. O'Connor AM, Fiset V, DeGrasse C, Graham ID, Evans W, Stacey D, et al. Decision aids for patients considering options affecting cancer outcomes: evidence of efficacy and policy implications. J Natl Cancer Inst Monogr 1999(25):67-80.

15. Jimison HB, Sher PP. Decision Support for Patients. In: Berner ES, editor. Clinical Decision Support Systems Theory and Practice. New York: Springer-Verlag; 1999. p. 139-66.

16. Comprehensive Health Enhancement Support System (CHESS). 2008 [cited 2008 Nov 12]; Available from: https://chess.wisc.edu/chess/home/ home.aspx.

17. Database of Individual Patient Experiences (DIPEx) 2008 [cited 2008 Nov 12]; Available from: http:// www.dipex.org/.

18. Foundation for Informed Medical Decision Making. 2008 [cited 2008 Nov 12]; Available from: http://www.informedmedicaldecisions.org/.

19. Mayo Clinic. 2008 [cited 2008 Nov 12]; Available from: http://mayoclinic.com/.

20. NexCura. 2008 [cited 2008 Nov 12]; Available from: http://www.nexcura.com/.

21. Schwitzer G. A Review of Features in Internet Consumer Health Decision-support Tools. J Med Internet Res 2002;4(2):e11.

22. Christensen H, Griffiths KM, Jorm AF. Delivering interventions for depression by using the internet: randomised controlled trial. BMJ 2004 January 31 , 2004:328(7434):265.

23. Fung V, Ortiz E, Huang J, Fireman B, Miller R, Selby JV, et al. Early experiences with e-health services (1999-2002): promise, reality, and implications. Med Care 2006 May;44(5):491-6.

24. Ralston JD, Carrell D, Reid R, Anderson M, Moran M, Hereford J. Patient web services integrated with a shared medical record: patient use and satisfaction. J Am Med Inform Assoc 2007 NovDec;14(6):798-806.

25. Frost HJ, Massagli PM. Social Uses of Personal Health Information Within PatientsLikeMe, an Online Patient Community: What Can Happen When Patients Have Access to One Another's Data. J Med Internet Res 2008;10(3):e15.

26. Weingart SN, Rind D, Tofias Z, Sands DZ. Who Uses the Patient Internet Portal? The PatientSite Experience. J Am Med Inform Assoc 2006 January 1, 2006;13(1):91-5.

27. Tang PC, Black W, Buchanan J, Young CY, Hooper D, Lane SR, et al. PAMFOnline: integrating EHealth with an electronic medical record system. AMIA Annu Symp Proc 2003:644-8.

28. The International Medical Informatics Association (IMIA) Web 2.0 Taskforce Portal. 2008 [cited 2008 Nov 12]; Available from: http://www.differanceengine.net/imia20/.

29. The International Society for Research on Internet Interventions. 2008 [cited 2008 Nov 12]; Available from: http://www.isrii.org/.

30. Murray PJ, Cabrer M, Hansen M, Paton C, Elkin PL, Erdley WS. Towards addressing the opportunities and challenges of Web 2.0 for health and informatics. Yearb Med Inform 2008:44-51.

31. Ottawa Health Research Institute. 2008 [cited 2008 
Nov 12]; Available from: http://decisionaid.ohri.ca/ 32. International Patient Decision Aid Standards (IPDAS) Collaboration. 2008 [cited 2008 Nov 12]; Available from: http://ipdas.ohri.ca/.

33. Elwyn G, O'Connor A, Stacey D, Volk R, Edwards A, Coulter A, et al. Developing a quality criteria framework for patient decision aids: online international Delphi consensus process. BMJ 2006 Aug 26;333(7565):417.

34. Cochrane Decision Aid Registry. 2008 [cited 2008 Nov 12]; Available from: http://decisionaid.ohri.ca/ cochinvent.php.

35. O'Connor AM, Rostom A, Fiset V, Tetroe J, Entwistle V, Llewellyn-Thomas H, et al. Decision aids for patients facing health treatment or screening decisions: systematic review. BMJ 1999 Sep 18:319(7212):731-4.

36. Frosch DL, Bhatnagar V, Tally S, Hamori CJ, Kaplan RM. Internet Patient Decision Support: A Randomized Controlled Trial Comparing Alternative Approaches for Men Considering Prostate Cancer Screening. Arch Intern Med 2008 February 25, 2008;168(4):363-9.

37. O'ConnorAM. Validation of a decisional conflict scale. Med Decis Making 1995 Jan-Mar;15(1):25-30.

38. Janis IL, Mann L. Decision making. New York: The Free Press; 1977.

39. O'Connor AM, Drake ER, Wells GA, Tugwell P, Laupacis A, Elmslie T. A survey of the decisionmaking needs of Canadians faced with complex health decisions. Health Expect 2003 Jun;6(2):97-109.

40. Lau AYS, Coiera EW. Do People Experience Cognitive Biases while Searching for Information? J Am Med Inform Assoc 2007 September 1, 2007;14(5):599-608.

41. Lau AYS, Coiera EW. Can Cognitive Biases during Consumer Health Information Searches Be Reduced to Improve Decision Making? J Am Med Inform Assoc 2009 January 1, 2009;16(1):54-65.

42. Coiera E. The Internet's challenge to health care provision. BMJ 1996 January 6, 1996;312(7022):3-4.

43. Butte AJ. Translational Bioinformatics: Coming of Age. J Am Med Inform Assoc 2008 NovDec;15(6):709-14.

44. Emery J, Hayflick S. The challenge of integrating genetic medicine into primary care. BMJ 2001;322(7293):1027-30.

45. Benson DA, Karsch-Mizrachi I, Lipman DJ, Ostell J, Wheeler DL. GenBank. Nucleic Acids Res 2007 Jan;35(Database issue):D21-D5.

46. Wheeler DL, Barrett T, Benson DA, Bryant SH, Canese K, Chetvernin V, et al. Database resources of the National Center for Biotechnology Information. Nucleic Acids Res 2008 Jan;36(Database issue):D13-D21.

47. Baars MJH, Henneman L, Leo P. Deficiency of knowledge of genetics and genetic tests among general practitioners, gynecologists, and pediatricians: A global problem. Genetics in Medicine 2005;7(9):605.

48. Burke W, Emery J. Genetics education for primarycare providers. Nat Rev Genet 2002;3(7):561-6.

49. Metcalfe S, Hurworth R, Newstead J, Robins R Needs assessment study of genetics education for general practitioners in Australia. Genetics in Medicine 2002;4(2):71.

50. Scheuner MT, Sieverding P, Shekelle PG. Delivery of genomic medicine for common chronic adult diseases: a systematic review. JAMA 2008 Mar 19;299(11):1320-34.

51. Alizadeh AA, Eisen MB, Davis RE, Ma C, Lossos IS, Rosenwald A, et al. Distinct types of diffuse large B-cell lymphoma identified by gene expression profiling. Nature 2000;403(6769):503-11.

52. Straus SE. Individualizing treatment decisions. The likelihood of being helped or harmed. Eval Health Prof 2002 Jun;25(2):210-24

53. Taube J, Halsall D, Baglin T. Influence of cytochrome P-450 CYP2C9 polymorphisms on warfarin sensitivity and risk of over-anticoagulation in patients on long-term treatment. Blood. 2000;96(5):1816.

54. Hylek EM, Heiman H, Skates SJ, Sheehan MA, Singer DE. Acetaminophen and other risk factors for excessive warfarin anticoagulation. JAMA. 1998 Mar;279(9):657-62.

55. Kearon C, Ginsberg JS, Kovacs MJ, Anderson DR, Wells P, Julian JA, et al. Comparison of low-intensity warfarin therapy with conventional-intensity warfarin therapy for long-term prevention of recurrent venous thromboembolism. N Engl J Med 2003 Aug;349(7):631-9.

56. Reynolds KK, Valdes Jr R, Hartung BR, Linder MW. Individualizing warfarin therapy. Personalized Medicine 2007;4(1):11-31.

57. Schwarz UI, Ritchie MD, Bradford Y, Li C, Dudek SM, Frye-Anderson A, et al. Genetic determinants of response to warfarin during initial anticoagulation. N Engl J Med 2008 Mar 6;358(10):999-1008.

58. Anderson JL, Horne BD, Stevens SM, Grove AS, Barton S, Nicholas ZP, et al. Randomized trial of genotype-guided versus standard warfarin dosing in patients initiating oral anticoagulation. Circulation 2007 Nov 27;116(22):2563-70.

59. FDA Approves Updated Warfarin (Coumadin) Prescribing Information.. Available from: http:// www.fda.gov/bbs/topics/news/2007/ new01684.html

60. McWilliam A, Lutter RW, Nardinelli C, for Regulatory Studies AEIBJC. Health Care Savings from Personalizing Medicine Using Genetic Testing: The Case of Warfarin: AEI-Brookings Joint Center for Regulatory Studies; 2006.

61. Weinstein RA. Nosocomial infection update. Emerg Infect Dis 1998;4(3):416-20

62. Wenzel RP, Pfaller MA. Feasible and desirable future targets for reducing the costs of hospital infections. J Hosp Infect 1991 Jun;18 Suppl A:94-8.

63. Ibrahim EH, Sherman G, Ward S, Fraser VJ, Kollef $\mathrm{MH}$. The influence of inadequate antimicrobial treatment of bloodstream infections on patient outcomes in the ICU setting. Chest $2000 \mathrm{Jul} ; 118(1): 146-55$.

64. Playford EG, Craig JC, Iredell JR. Carbapenemresistant Acinetobacter baumannii in intensive care unit patients: risk factors for acquisition, infection and their consequences. J Hosp Infect 2007 Mar;65(3):204-11.

65. Evans RS, Pestotnik SL, Classen DC, Clemmer TP, Weaver LK, Orme JF, et al. A computer-assisted management program for antibiotics and other antiinfective agents. N Engl J Med 1998 Jan;338(4):232-8.

66. Paul M, Andreassen S, Tacconelli E, Nielsen AD, Almanasreh N, Frank U, et al. Improving empirical antibiotic treatment using TREAT, a computerized decision support system: cluster randomized trial.
JAntimicrob Chemother 2006 Dec;58(6):1238-45

67. Thursky KA, Buising KL, Bak N, MacGregor L, Street AC, MacIntyre CR, et al. Reduction of broadspectrum antibiotic use with computerized decision support in an intensive care unit. Int J Qual Health Care 2006 Jun;18(3):224-31.

68. Yu VL, Fagan LM, Wraith SM, Clancey WJ, Scott AC, Hannigan J, et al. Antimicrobial selection by a computer. A blinded evaluation by infectious diseases experts. JAMA 1979 Sep;242(12):1279-82.

69. Thursky K. Use of computerized decision support systems to improve antibiotic prescribing. Expert Rev Anti Infect Ther 2006 Jun;4(3):491-507.

70. Partridge SR, Tsafnat G, Coiera E, Iredell JR. Gene cassettes and cassette arrays in mobile resistance integrons. FEMS Microbiol Rev 2009; under review, accepted in principle.

71. Young J, Stevenson KB. Real-time surveillance and decision support: Optimizing infection control and antimicrobial choices at the point of care. Am J Infect Control. 2008:36:S67-74.

72. Moore KM, Edgar BL, McGuinness D. Implementation of an automated, real-time public health surveillance system linking emergency departments and health units: rationale and methodology. CJEM 2008 Mar;10(2):114-9.

73. Williams CJ, Schenkel K, Eckmanns T, Altmann D, Krause G. FIFA World Cup 2006 in Germany: enhanced surveillance improved timeliness and detection. Epidemiol Infect 2008 Jul 31:1-9.

74. Wu TS, Shih FY, Yen MY, Wu JS, Lu SW, Chang $\mathrm{KC}$, et al. Establishing a nationwide emergency department-based syndromic surveillance system for better public health responses in Taiwan. BMC Public Health 2008;8:18.

75. Zheng W, Aitken R, Muscatello DJ, Churches T. Potential for early warning of viral influenza activity in the community by monitoring clinical diagnoses of influenza in hospital emergency departments. BMC Public Health 2007:7:250.

76. Davis XM, MacDonald S, Borwein S, Freedman DO, Kozarsky PE, von Sonnenburg F, et al. Health risks in travelers to China: the GeoSentinel experience and implications for the 2008 Beijing Olympics. Am J Trop Med Hyg 2008 Jul;79(1):4-8.

77. Gundlapalli AV, Olson J, Smith SP, Baza M, Hausam RR, Eutropius LJ, et al. Hospital electronic medical record-based public health surveillance system deployed during the 2002 Winter Olympic Games. Am J Infect Control 2007 Apr;35(3):163-71.

78. Meynard JB, Chaudet H, Texier G, Ardillon V, Ravachol F, Deparis X, et al. Value of syndromic surveillance within the Armed Forces for early warning during a dengue fever outbreak in French Guiana in 2006. BMC Med Inform Decis Mak 2008;8:29.

79. Buckeridge DL, Okhmatovskaia A, Tu S, O’Connor M, Nyulas C, Musen MA. Understanding detection performance in public health surveillance: modeling aberrancy-detection algorithms. J Am Med Inform Assoc 2008 Nov-Dec;15(6):760-9.

80. Mellmann A, Friedrich AW, Rosenkotter N, Rothganger J, Karch H, Reintjes R, et al. Automated DNA sequence-based early warning system for the detection of methicillin-resistant Staphylococcus aureus outbreaks. PLoS Med 2006 Mar;3(3):e33.

81. Shen Y, Adamou C, Dowling JN, Cooper GF. Estimating the joint disease outbreak-detection time 
when an automated biosurveillance system is augmenting traditional clinical case finding. J Biomed Inform 2008 Apr;41(2):224-31.

82. Brownstein JS, Freifeld CC, Reis BY, Mandl KD. Surveillance Sans Frontieres: Internet-based emerging infectious disease intelligence and the HealthMap project. PLoS Med 2008 Jul $8 ; 5(7): \mathrm{e} 151$.

83. Freifeld CC, Mandl KD, Reis BY, Brownstein JS HealthMap: global infectious disease monitoring through automated classification and visualization of Internet media reports. J Am Med Inform Assoc 2008 Mar-Apr;15(2):150-7.

84. Scotch M, Yip KY, Cheung KH. Development of grid-like applications for public health using Web 2.0 mashup techniques. J Am Med Inform Assoc 2008 Nov-Dec;15(6):783-6.

85. Cooper DL, Smith GE, Regan M, Large S, Groenewegen PP. Tracking the spatial diffusion of influenza and norovirus using telehealth data: a spatiotemporal analysis of syndromic data. BMC Med 2008;6:16

86. Kukafka R, Ancker JS, Chan C, Chelico J, Khan S, Mortoti S, et al. Redesigning electronic health record systems to support public health. J Biomed Inform 2007 Aug;40(4):398-409.

87. Rosen-Zvi M, Altmann A, Prosperi M, Aharoni E, Neuvirth H, Sonnerborg A, et al. Selecting antiHIV therapies based on a variety of genomic and clinical factors. Bioinformatics $2008 \mathrm{Jul}$ 1;24(13):i399-406

88. Tang J, Tao J, Urakawa H, Corander J. T-BAPS: a Bayesian statistical tool for comparison of microbial communities using terminal-restriction fragment length polymorphism (T-RFLP) data. Stat Appl Genet Mol Biol 2007;6:Article30.

89. Sintchenko V, Gallego B, Chung G, Coiera E. Towards bioinformatics assisted infectious disease control. BMC Bioinformatics 2008 Dec;10(S10).

90. Liu TF, Shafer RW. Web resources for HIV type 1 genotypic-resistance test interpretation. Clin Infect Dis 2006 Jun 1:42(11):1608-18

91. Moore M, Chan E, Lurie N, Schaefer AG, Varda DM, Zambrano JA. Strategies to improve global influenza surveillance: a decision tool for policymakers. BMC Public Health 2008;8:186.

92. Reis BY, Kirby C, Hadden LE, Olson K, McMurry AJ, Daniel JB, et al. AEGIS: a robust and scalable real-time public health surveillance system. J Am Med Inform Assoc 2007 Sep-Oct;14(5):581-8.

93. Gallego B, Sintchenko V, Wang Q, Hiley L, Gilbert GL, Coiera E. Biosurveillance of emerging biothreats using scalable genotype clustering. Journal of Biomedical Informatics 2009;42(1):66-73

94. Eslami S, de Keizer NF, Abu-Hanna A. The impact of computerized physician medication order entry in hospitalized patients - a systematic review. Int J Med Inform 2008 Jun;77(6):365-76.

95. Ash JS, Berg M, Coiera E. Some Unintended Consequences of Information Technology in Health Care: The Nature of Patient Care Information Systemrelated Errors. JAm Med Inform Assoc 2004 March 1, 2004;11(2):104-12.

96. Ammenwerth E, Schnell-Inderst P, Machan C, Siebert U. The Effect of Electronic Prescribing on Medication Errors and Adverse Drug Events: A
Systematic Review. J Am Med Inform Assoc 2008 September-October;15(5):585-600.

97. The Joint Commission. 2008 [cited 2008 Dec 15]; Available from: www.jointcommission.org/ SentinelEvents/SentinelEventAlert/sea_42.htm.

98. Wang CJ, Marken RS, Meili RC, Straus JB, Landman AB, Bell DS. Functional Characteristics of Commercial Ambulatory Electronic Prescribing Systems: A Field Study. J Am Med Inform Assoc 2005 May 1, 2005;12(3):346-56

99. Gaikwad R, Sketris I, Shepherd M, Duffy J. Evaluation of accuracy of drug interaction alerts triggered by two electronic medical record systems in primary healthcare. Health Informatics J 2007 Sep;13(3):163-77.

100. Mille F, Schwartz C, Brion F, Fontan JE, Bourdon O, Degoulet P, et al. Analysis of overridden alerts in a drug-drug interaction detection system. Int J Qual Health Care 2008 Dec;20(6):400-5.

101. Weingart SN, Toth M, Sands DZ, Aronson MD, Davis RB, Phillips RS. Physicians' decisions to override computerized drug alerts in primary care. Arch Intern Med 2003 Nov 24; 163(21):2625-31.

102. KoY, Abarca J, Malone DC, Dare DC, Geraets D, Houranieh A, et al. Practitioners' views on computerized drug-drug interaction alerts in the VA system. J Am Med Inform Assoc 2007 JanFeb;14(1):56-64.

103. Spina JR, Glassman PA, Belperio P, Cader R, Asch S. Clinical relevance of automated drug alerts from the perspective of medical providers. Am J Med Qual 2005 Jan-Feb;20(1):7-14.

104. van der Sijs H, Aarts J, Vulto A, Berg M. Overriding of drug safety alerts in computerized physician order entry. J Am Med Inform Assoc 2006 Mar-Apr;13(2):138-47.

105. Grizzle AJ, Mahmood MH, Ko Y, Murphy JE, Armstrong EP, Skrepnek GH, et al. Reasons provided by prescribers when overriding drug-drug interaction alerts. Am J Manag Care 2007 Oct:13(10):573-8.

106. Lin CP, Payne TH, Nichol WP, Hoey PJ, Anderson CL, Gennari JH. Evaluating Clinical Decision Support Systems: Monitoring CPOE Order Check Override Rates in the Department of Veterans Affairs' Computerized Patient Record System. J Am Med Inform Assoc 2008 SepOct;15(5):620-6.

107. Walker JM, Carayon P, Leveson N, Paulus RA, Tooker J, Chin H, et al. EHR safety: the way forward to safe and effective systems. J Am Med Inform Assoc 2008 May-Jun;15(3):272-7.

108. Ash JS, Sittig DF, Campbell EM, Guappone KP, Dykstra RH. Some unintended consequences of clinical decision support systems. AMIA Annu Symp Proc 2007:26-30

109. Coiera EW, Westbrook JI. Should clinical software be regulated? Med J Aust 2006 Jun 19;184(12):600-1.

110. US Certification Commission for Healthcare IT. 2008 [cited 2008 Dec 15]; Available from: www.cchit.org.

111. Leapfrog issues caution that quality assurance is necessary during implementation of computerized medication systems. 2008 [cited 2008 Dec
15]; Available from: www.leapfroggroup.org.

112. UK NHS Connecting for Health Initiative. 2008 [cited 2008 Dec 15]; Available from: www.connecting forhealth.nhs.uk systemsandservices/eprescribing/news/hazard.

113. Kilbridge PM, Classen DC. The informatics opportunities at the intersection of patient safety and clinical informatics. J Am Med Inform Assoc 2008 Jul-Aug;15(4):397-407.

114. Cacciabue PC, Vella G. Human factors engineering in healthcare systems: The problem of human error and accident management. Int J Med Inform 2008 Dec 18.

115. Paterno MD, Maviglia SM, Gorman PN, Seger DL, Yoshida E, Seger AC, et al. Tiering drugdrug interaction alerts by severity increases compliance rates. J Am Med Inform Assoc 2009 JanFeb;16(1):40-6.

116. Tamblyn R, Huang A, Taylor L, Kawasumi Y, Bartlett G, Grad R, et al. A randomized trial of the effectiveness of on-demand versus computer-triggered drug decision support in primary care. JAm Med Inform Assoc 2008 Jul-Aug;15(4):430-8.

117. Lo HG, Matheny ME, Seger DL, Bates DW, Gandhi TK. Impact of non-interruptive medication laboratory monitoring alerts in ambulatory care. J Am Med Inform Assoc 2009 JanFeb;16(1):66-71.

118. van der Sijs H, Aarts J, van Gelder T, Berg M, Vulto A. Turning off frequently overridden drug alerts: limited opportunities for doing it safely. J Am Med Inform Assoc 2008;15(4):439-48.

119. Graham TA, Kushniruk AW, Bullard MJ, Holroyd BR, Meurer DP, Rowe BH. How usability of a web-based clinical decision support system has the potential to contribute to adverse medical events. AMIA Annu Symp Proc 2008:257-61.

120. Magrabi F. Using cognitive models to evaluate safety-critical interfaces in healthcare. CHI'08 extended abstracts on Human factors in computing systems; Florence, Italy: ACM; 2008.

121. Lamy JB, Duclos C, Bar-Hen A, Ouvrard P, Venot A. An iconic language for the graphical representation of medical concepts. BMC Med Inform Decis Mak. 2008;8:16.

122. Zheng K, Padman R, Johnson MP, Diamond HS. An Interface-Driven Analysis of User Interactions with an Electronic Health Records System. J Am Med Inform Assoc 2008 Dec 11

123. Bonnabry P, Despont-Gros C, Grauser D, Casez P, Despond M, Pugin D, et al. A risk analysis method to evaluate the impact of a computerized provider order entry system on patient safety. J Am Med Inform Assoc 2008 Jul-Aug;15(4):453-60.

124. Magrabi F, McDonnell G, Westbrook JI, Coiera E. Using an accident model to design safe electronic medication management systems. Medinfo 2007;12(Pt 2):948-52.

\section{Correspondence to}

Enrico Coiera

Centre for Health Informatics

University of New South Wales

UNSW 2052 NSW Australia

E-mail: e.coiera@unsw.edu.au 